Rev. Biol. Trop., 47(4): 1081-1086, 1999

www.ucr.ac.cr www.ots.ac.cr www.ots.duke.edu

\title{
Alimentación de Microthrissa congica (Osteichthyes: Clupeidae) en la cuenca alta del río Congo (África)
}

\author{
Godefroid Mambyanga Mokoma $^{1 *}$, Luc Devos y Luis Cardona ${ }^{2}$
}

1 Département d'Ecologie et Conservation de la Nature, Faculté des Sciences, Université de Kisangani, B.P. 2012 Kisangani, République Démocratique du Congo ex Zaire.

2 Departamento de Biología Animal, Facultad de Biología, Universidad de Barcelona, Avda. Diagonal 645, 08028Barcelona, España

* Dirección actual : Departamento de Biología Animal, Facultad de Biología, Universidad de Barcelona, Avda. Diagonal 645, 08028-Barcelona, España. Fax : +34-934035740. Correo electrónico :mokoma@porthos.bio.ub.es

Recibido 12-I-1999. Corregido 8-VII-1999. Aceptado 11-VIII-1999

\begin{abstract}
The feeding habits of the fish Microthrissa congica were studied for a year in the upper Congo river basin, by monthly analysis of stomach contents of adult specimens $(\mathrm{N}=460)$. The nymphs of chironomid midges represented the bulk of the diet throughout most of the year, although terrestrial insects became more abundant in the rainy season. Niche breadth also increased in the wet season. This species feeds mainly in the water column and benthos is not an important food source.
\end{abstract}

Key words: Feeding habits, Africa, Microthrissa congica, Clupeidae, insectivores, Congo river.

La mayoría de los estudios sobre la ictiofauna de la cuenca del Río Congo son de carácter sistemático. En cambio, existe poca información sobre la biología de la mayoría de las especies presentes en la región.

La familia Clupeidae está representada en la zona por Pellonula leonensis, Odaxothrissa losera y cinco especies del género $\mathrm{Mi}$ crothrissa (Poll 1974, Gourène 1988, Gourène \& Teugels 1989, 1994). El río Tshopo, afluente de la parte alta de la cuenca, está habitado por $O$. losera, Microthrissa royauxi y Microthrissa congica. Esta última es la especie más abundante y posee importancia comercial (Mokoma y Devos observación personal, 1989). Se trata de una especie de pequeño tamaño, endémica del Río Congo y distribuida por toda su cuenca (Gourène 1988). Apenas existe información previa sobre su biología, aunque Matthes (1964) la considera gregaria.
El objetivo de esta investigación es determinar los hábitos alimentarios de Microthrissa congica (Regan, 1917) en el curso superior de la cuenca del río Congo.

\section{MATERIALES Y MÉTODOS}

Los ejemplares se capturaron en una zona del río Tshopo $\left(0^{\circ} 31^{\prime} \mathrm{N}\right.$ y $\left.25^{\circ} 11^{\prime} \mathrm{E}\right)$ situada a una altitud media de 401 m.s.m., dentro del municipio de Kisangani. El clima es ecuatorial, cálido durante todo el año y con una estación seca y otra lluviosa (Fig. 1).

Entre mayo de 1988 y abril de 1989 se capturaron un total de 460 ejemplares de $M$. congica utilizando tres trasmallos de $30 \times 1.5 \mathrm{~m}$ y mallas de 12,15 y $20 \mathrm{~mm}$ de nudo a nudo respectivamente. Las capturas fueron mensuales y las redes se calaron 24 horas en la misma localidad. Los ejemplares se conservaron en formol al $4 \%$. 
En el laboratorio, los especímenes se midieron y pesaron antes de extraerles el tubo digestivo. El factor de condición se determinó mediante la ecuación

$$
\mathrm{Fc}=100 \mathrm{Pc} / \mathrm{L}^{3}
$$

donde Fc es el factor de condición, Pc es el peso de la carcasa del espécimen sin las gónadas expresado en $\mathrm{g}$ y $\mathrm{L}$ es la longitud estándar del espécimen expresada en cm (Busacker et al. 1990).

También se calculó el índice gonadosomático mediante la ecuación

$$
\text { IGS }=100 \mathrm{Pg} / \mathrm{P}
$$

donde IGS es el índice gonadosomático, Pg es el peso de las gónadas y $\mathrm{P}$ es el peso total del animal.

El contenido estomacal se vertió en una cápsula de Petri y posteriormente se analizó en un estéreomicroscopio de disección. Las presas se identificaron hasta el taxon más bajo posible con la ayuda de las claves de Roth (1980) y Tachet et al. (1980).

Para evaluar la importancia de cada grupo de presas en la alimentación de $M$. congica se emplearon dos métodos diferentes. La frecuencia de ocurrencia en los estómagos de cada categoría se calculó de acuerdo con la ecuación

$$
\mathrm{Fa} i=100 \mathrm{n} i / \mathrm{N}
$$

donde Fai es la frecuencia de aparición de la categoría $i$, ni es el número de estómagos donde apareció dicha especie y $\mathrm{N}$ es el número total de estómagos estudiados (Hureau 1970). Este método ofrece una buena estimación de las preferencias alimentarias de la especie, pero no indica nada sobre la importancia energética de cada categoría de presas (Hyslop 1980)

Con el fin de complementar dicha información, las presas se dividieron en sèis grandes categorías (insectos acuáticos, insectos terrestres, arañas, crustáceos, alevines y detritus indeterminados), con el fin de acumular suficiente material para poder ser pesado. Para cada una de ellas, se calculó su importancia por- centual en el conjunto de la masa del contenido estomacal, de acuerdo con la ecuación

$$
\mathrm{Fp} i=100 \mathrm{p} i / \mathrm{P}
$$

donde Fpi es la importancia porcentual de la categoría de presas $i$, pi es la masa de las presas de la cateogría $i$ y $\mathrm{P}$ es la masa total del contenido estomacal.

A continuación, se calculó el índice alimentario de Lauzanne de acuerdo con la ecuación dada por Lauzanne (1976),

$$
\mathrm{I} a i=(\mathrm{Fa} i \mathrm{Fp} i) / 100
$$

donde Ia $i$ es la importancia de la categoría $i$ en la dieta, Fa $i$ es su frecuencia de aparición y Fp $i$ su importancia porcentual.

Las frecuencias de aparición fueron utilizadas para calcular la amplitud del nicho, de acuerdo con el índice de Gladfelter-Jonhson modificado por Cardona (1991), definido como

$$
\mathrm{b}=(\mathrm{SFa} i-\mathrm{s}) / 100 \mathrm{R}
$$

donde (b es la amplitud del nicho, Fai es la frecuencia de aparición de la presa $i$, s(es la desviación típica de las frecuencias de aparición y $\mathrm{R}$ es el número total de categoría de presas.

La existencia de diferencias estadísticas entre los valores mensuales de Fc y de IGS se midió mediante la prueba ANOVA seguida de la prueba de Scheffe. Para comprobar si existía relación alguna entre los cambios ambientales y la dieta, se hizo un análisis de correlación entre tres parámetros ambientales (temperatura, precipitaciones y precipitaciones del mes anterior), la amplitud del nicho y la frecuencia de ocurrencia de los diferentes grupos de presas. Para ello se empleó el coeficiente de correlación no paramétrico de Kendall.

\section{RESULTADOS}

Se capturaron un total de 460 ejemplares de $M$. congica, con una longitud estándar media de $69.35 \pm 4.06 \mathrm{~mm}$ (talla mínima: $55 \mathrm{~mm}$; 

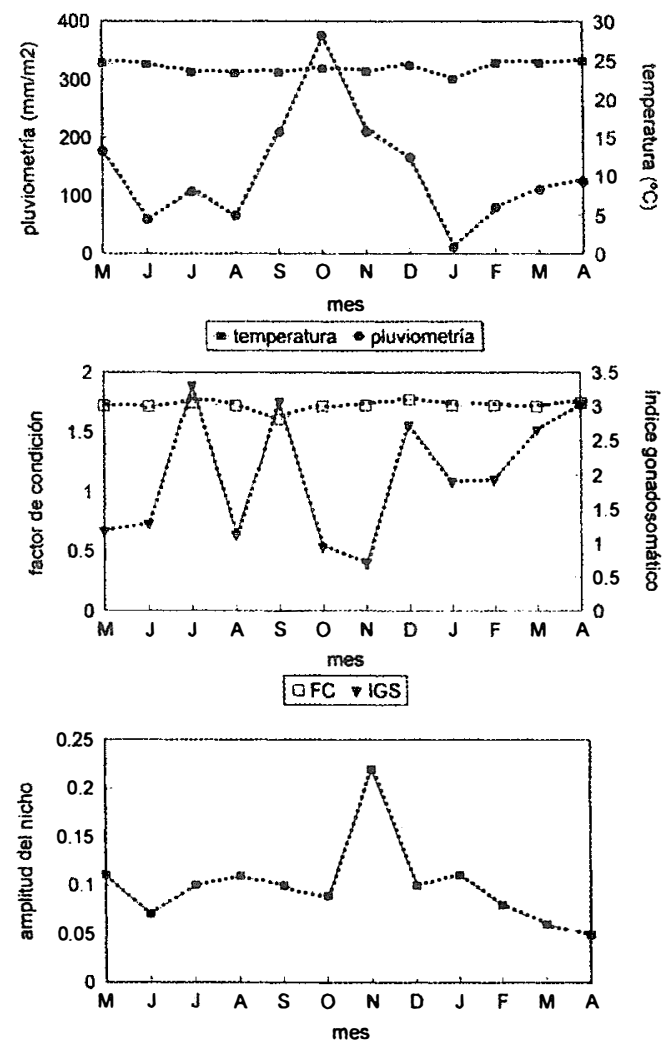

Fig. 1 : Evolución mensual de la temperatura y de la pluviometría en Kisangani (arriba), evolución mensual del factor de condición y del índice gonadosomático de $\mathrm{Mi}$ crothrissa congica en el río Tshopo (centro) y evolución mensual de la amplitud del nicho trófico de Microthrissa congica en el río Tshopo (abajo).

talla máxima: $79 \mathrm{~mm}$ ). La presencia de gónadas demostró que se trataba en todos casos de ejemplares adultos. El peso medio del cuerpo del animal, sin las gónadas, fue de $5.84 \pm 0.86$ $\mathrm{g}$, sin que existieran variaciones significativas a lo largo del estudio (ANOVA; p>0.05). El factor de condición tampoco presentó variaciones significativas a lo largo del estudio, siendo el valor medio de $1.71 \pm 0.022$ (ANOVA; $\mathrm{p}>0.05$ ) (Fig. 1). El índice gonadosomático presentó acusadas oscilaciones y los valores registrados durante los meses de julio, septiembre, diciembre, marzo y abril fueron significativamente superiores a los registrados el resto del año (ANOVA; p<0.05) (Fig. 1).

El Cuadro 1 muestra las frecuencias de aparición mensuales de las diferentes cate- gorías de presas. Las presas más importantes fueron las ninfas de quironómidos, al aparecer en más del $50 \%$ de los estómagos, especialmente durante la estación seca. En segundo y tercer orden de importancia están los himenópteros y los detritus indeterminados, que aparecen en todos los meses pero con frecuencia de aparición muy variables. Los isópteros y las larvas de plecópteros, coleópteros, efemerópteros y quironómidos únicamente fueron consumidos durante la época lluviosa. Las arañas, las larvas de tricópteros, los ortópteros, los crustáceos y los alevines de $M$. congi$c a$ aparecieron de forma esporádica en el contenido estomacal.

Considerando el conjunto de la información disponible, se observa que las ninfas de quironómidos constituyen la base de la dieta, la cual se complementa con himenópteros, larvas de efemerópteros y de quironómidos y detritus. Las restantes presas son incidentales.

Combinando la frecuencia de ocurrencia de los seis grupos principales de presas con su importancia porcentual (Cuadro 2), se observa que el grueso de la dieta corresponde a los insectos acuáticos, seguidos por los insectos terrestres.

Como consecuencia, la amplitud del nicho es muy pequeña, oscilando entre 0.05 y 0.22 (Fig. 1). Este fenómeno se acrecienta durante la estación seca, entre de enero y septiembre, cuando disminuye el nivel del río y $M$. congi$c a$ se alimenta casi exclusivamente de insectos acuáticos (ninfas de quironómidos). Durante la estación lluviosa, entre octubre y diciembre, el nivel del río aumenta notablemente, por lo que el bosque adyacente se inunda y $M$. congica amplia el espectro de presas consumidas, incorporando grandes cantidades de himenópteros y detritus indeterminados.

A pesar de ello, la correlación entre los parámetros ambientales y los descriptores de la dieta fue muy baja o no existió. Las mejores correlaciones se hallaron entre la lluvia del mes anterior y los siguientes parámetros: amplitud del nicho $(\mathrm{K}=0.254 ; \mathrm{p}>0.05)$, frecuencia de aparición de isópteros $(\mathrm{K}=0.3045$; $\mathrm{p}>0.05)$, frecuencia de aparición de ninfas de quironómidos 


\section{CUADRO 1}

Evolución mensual de la frecuencia de aparición de las presas consumidas por Microthrissa congica. m indica la media anual.

\begin{tabular}{|c|c|c|c|c|c|c|c|c|c|c|c|c|c|}
\hline Categoría de presas & $\mathbf{M}$ & $\mathbf{J}$ & $\mathrm{JL}$ & A & $\mathrm{S}$ & $\mathrm{O}$ & $\mathbf{N}$ & D & $\mathrm{E}$ & $\mathrm{F}$ & $\mathbf{M}$ & A & $\mathrm{m}$ \\
\hline Ninfas de quironómidos & 59 & 76 & 4 & 90 & 72 & 50 & 13 & 3 & 22 & 60 & 80 & 30 & 53 \\
\hline Larvas de quironómidos & 50 & 6 & 0 & 53 & 6 & 58 & 0 & 3 & 7 & 67 & 0 & 0 & 15 \\
\hline Larvas de tricópteros & 0 & 0 & 0 & 0 & 0 & 0 & 25 & 0 & 0 & 0 & 0 & 0 & 1 \\
\hline Larvas de efemerópteros & 0 & 0 & 85 & 3 & 0 & 0 & 38 & 3 & 44 & 7 & 0 & 0 & 15 \\
\hline Larvas de plecópteros & 0 & 0 & 4 & 0 & 3 & 0 & 0 & 25 & 11 & 0 & 4 & 4 & 3 \\
\hline Larvas de odonatos & 9 & 6 & 4 & 13 & 0 & 17 & 25 & 6 & 4 & 0 & 0 & 0 & 6 \\
\hline Coleópteros & 5 & 0 & 4 & 0 & 6 & 0 & 25 & 6 & 22 & 13 & 0 & 7 & 7 \\
\hline Himenópteros & 27 & 12 & 30 & 17 & 22 & 8 & 75 & 38 & 30 & 7 & 28 & 4 & 24 \\
\hline Isópteros & 9 & 0 & 0 & 7 & 3 & 17 & 63 & 6 & 0 & 7 & 0 & 11 & 7 \\
\hline Ortópteros & 0 & 0 & 0 & 0 & 25 & 8 & 0 & 0 & 15 & 0 & 0 & 0 & 5 \\
\hline Dípteros terrestres & 0 & 0 & 0 & 3 & 13 & 0 & 0 & 0 & 0 & 0 & 0 & 0 & 2 \\
\hline Detritus & 31 & 41 & 33 & 16 & 34 & 5 & 100 & 78 & 37 & 40 & 0 & 25 & 40 \\
\hline Crustáceos & 0 & 0 & 0 & 3 & 0 & 0 & 0 & 0 & 0 & 0 & 0 & 0 & 1 \\
\hline Arañas & 0 & 0 & 4 & 0 & 0 & 0 & 0 & 0 & 0 & 0 & 0 & 0 & 1 \\
\hline Alevines & 0 & 0 & 7 & 0 & 0 & 0 & 0 & 0 & 0 & 0 & 0 & 4 & 1 \\
\hline
\end{tabular}

\section{CUADRO 2}

Frecuencia de aparición, importancia relativa ponderal y valor del índice alimentario de las seis categorías principales de presas de Microthrissa congica

Categoría de presas

Insectos acuáticos

Insectos terrestres

Alevines

Crustáceos

Arañas

Detritus
Frecuencia de aparición

100
100
1,11
0,4
0,4
37,4

( $\mathrm{K}=-0.2727 ; \mathrm{p}>0.05)$, frecuencia de aparición de himenópteros $(K=0.229 ; \mathrm{p}>0.05)$ y frecuencia de aparición de detritus $(\mathrm{K}=0.3030 ; \mathrm{p}>0.05)$.

\section{DISCUSIÓN}

Todos los clupéidos son filtradores (López 1966, James 1988). Las especies marinas capturan preferentemente crustáceos y fitoplancton (James 1988, Ortaz et al. 1996, Huse y Toresen 1996). La alimentación de las formas dulceacuícolas lacustres es similar (Coulter 1991), mientras las fluviales basan normalmente su alimentación en insectos (Lauzanne 1988; Grabe 1996). Los resultados del presente estudio se ajustan a dicho
Importancia ponderal

Indice alimentario

70,75
10,94
0,0003
0,0004
0,03
5,68

70,75

10,94

0,000003

0,0000016

0,00012

2,12

patrón, ya que $M$. congica consume básicamente insectos. A diferencia de algunas especies de clupéidos fluviales capaces de utilizar extensivamente los insectos bentónicos, este tipo de presa juega un papel muy poco importante en la dieta de la especie considerada, ya que ésta se muestra básicamente como una forma pelágica.

El canibalismo accidental, muy frecuente en otros clupéidos, es prácticamente insignificante en $M$. congica, pues la presencia de alevines en el contenido estomacal es muy baja. El desove parece producirse en el mismo río (Mokoma observación personal 1989), por lo que debe existir algún tipo de segregación espacial ontogénica para evitar el çonsumo accidental de los alevines. 
La variación estacional de la dieta y de la amplitud del nicho se ajusta a lo que suele suceder con las especies fluviales de las regiones tropicales (Zaret y Rand 1971, Lauzanne 1988), es decir, durante la época seca se reduce la amplitud del nicho como consecuencia de una reducción en la variedad de alimento disponible. Durante la época lluviosa, aumenta la utilización de presas de origen terrestre, principalmente isópteros, himenópteros y detritus, lo que provoca un aumento de la amplitud del nicho, así como un cierto descenso en la utilización de ninfas de quironómidos. Ahora bien, la disponibilidad de las presas terrestres depende de su arrastre hacia el río por el agua de escorrentía, por lo que la precipitación mensual no se correlaciona bien con la amplitud del nicho ni con la frecuencia de aparición de dichas presas. La correlación es mejor cuando se considera la precipitación del mes anterior, aunque sigue siendo muy baja.

$M$. congica no parece verse muy afectada por la reducción de la amplitud del nicho durante la época seca, aunque la obligue a alimentarse de forma casi exclusiva de ninfas de quironómidos. Durante este período, el factor de condición no se modifica y el índice gonadosomático alcanza valores elevados, lo que sugieren una reproducción activa.

Todo esto sugiere que la población de $M$. congica del río Tshopo tiene un carácter oportunista y pelágico. Ahora bien, como los artrópodos más abundantes en el plancton durante gran parte del año parecen ser las ninfas de quironómidos, cualquier fenómeno que afecte a su abundancia repercutirá negativamente en la pesquería de $M$. congica, siempre y cuando no aumente de forma paralela la disponibilidad de insectos terrestes. En cambio, el bosque circundante parece ser poco importante para la alimentación de este clupéido, si se mantiene la oferta de quironómidos.

\section{RESUMEN}

Mensualmente durante un año, se estudió la alimentación de Microthrissa congica en la cuenca alta del Río Congo, mediante el análisis de contenidos estomacales de ejemplares adultos. De acuerdo con la frecuencia de aparición, las ninfas de quironómidos constituyen la base de su alimentación durante todo el año. La importancia de los insectos de origen terrestre aumenta durante la época de lluvias, momento en que aumenta la amplitud del nicho. Estos resultados demuestran que este clupeido es una especie insectívora pelágica, utilizando muy poco el bentos como fuente de alimento.

\section{REFERENCIAS}

Busacker, G.P., I.R. Adelman \& E.M. Goolish. 1990. Growth. p. 363-387. In C.B. Schreck \& P.B. Moyle (eds.) Methods for fish biology. American Fisheries Society, Bethesda, Maryland.

Cardona, L.1991. Measurement of tropic niche breadth using occurence frequencies. J.Fish Biol. 39: 901903.

Coulter,G.W.1991. Lakes Tanganyika and its life. Natural History Museum Publications, Oxford University, Oxford. $345 \mathrm{p}$.

Gourène G., 1988. Révision systématique des Clupeidae d'eau douce de l'ouest et du centre africain. Morphologigie,Biométrie,Ostéologie et Zoogéographie des genres: Pellonula,Odaxothrissa, Cynothrissa, poecilothrissa et Microthrissa.Thèse de doctorat. Institut National Polytechnique de Toulouse, Francia. 270 p.

Gourène,G. \& G.G.Teugels.1989. Révision systématique du genre Microthrissa Boulanger,1902 des eaux douces africaines (pisces;Clupeidae).Rev.Hydrobiol. Trop.22: 129-156.

Gourène,G.\& G.G.Teugels. 1994. Synopsis de la classification et phylogénie des pellonulinae de l'Afrique Occidentale et Centrale (Teleostei;Clupeidae).J.Afr.Zool.108: 77-91.

Grabe, A.1996. Feeding chronology and habits of Alosa spp.(Clupeidae) juveniles from the lower Hudson $\mathrm{Ri}$ ver estuary,New- York.Env. Biol. Fish.47: 321-326.

Hureau,J.C.1970. Biologie comparée de quelques posissons antarcti- ques (Notothenidae). Bull. Inst. Oceanogr. Monaco $68: 1-224$

Huse,G \& R. Toresen.1996. A comparative study of the feeding habits of herring (Clupea harengus, Clupeidae) and Capelin (Mallotus villosus, Osmeridae, Müller) in the Barents Sea. Sarsia 81: 143-153.

Hyslop,E.J.1980. Stomach contents analysis. A review of methods and their application.J.Fish Biol. 19: 411-429. 
James,A.G.1988. Are Clupeids microphagist herbivorous or omnivorous? A review of the diets of some commercially important Clupeids. S.Af.J. Mar.Sci.7: 161-177.

Lauzanne, L. 1976. Régimes alimentaires et relations trophiques des poissons du lac Tchad. Cah. ORSTOM sér Hydrobiol. 10: 267-310.

Lauzanne,L.1988. Les habitudes alimentaires des poissons d'eau douce Africains. p. 221-242. In C. Lévêque, M.N. Bruton \& G.W. Ssentongo (eds.). Biologie et écologie des poissons d'eau douce Africains. ORSTOM. París.

López, J.1963. Peces emigrantes.Garriga, Barcelona. 260 p.

Matthes, H. 1964. Les poissons du lac Tumba et de la région Ikela. Étude systématique et écologique. Ann. Mus. Roy. Af. Cent. 126: 1-204.
Ortaz,M.,M. Rocha. \& M. Posada.1996. Food habits of the sympatric fishes Harengula humeralis and H.clupeola (Clupeidae) in the archipelago de los Roques National Park,Venezuela. J. Sci.32: 26-32.

Poll,M.1974. Synopsis et distribution géographique des Clupeidae africains, description de trois nouvelles espèces. Bull .Acad .Roy. Belg. 5: 141-161.

Roth,M.1980. Initiation à la morphologie,la Systématique et la Biologie des insectes. ORSTOM, París. 212 p.

Tachet H.,M. Bornaud. \& P. Richaux.1980. Introduction à l'étude des macroinvertébrés des eaux douces, (Systématique élémentaire et aperçu écologique).Université de Lion I, Lion, Francia. 150 p.

Zaret,T.M. \& E.S. Rand.1971. Competition in tropical stream fishes: Support for the competitive exclusion principle.Ecology 52: 143-160. 